\title{
Gas-powered jet injection compared with conventional methods of injection using lignocaine and technetium-99
}

\author{
E D COOKE, SALLY A BOWCOCK, A JOHNSTON, A T ELLIOTT
}

\section{Summary and conclusions}

Plasma concentrations of lignocaine and the clearance rates of technetium $-99^{\mathrm{m}}$ were compared in 10 healthy volunteers after injection with a gas-powered jet system and a needle and syringe. The dosage was highly accurate with jet injection. Bioavailability was comparable for both methods.

\section{Introduction}

The possibility of using a needleless, and hence painless, method of injection for therapeutic substances is attractive. Several needleless systems are available, but none are in general use. Med-E-Jet ${ }^{1}$ is a gas-powered and easily handled system that is likely to find wide usage, particularly for local anaesthesia. We have compared plasma concentrations and clearance rates using Med-E-Jet against the conventional method of a needle and syringe.

\section{Patients and methods}

The investigation was in two parts. (1) Five men and five women volunteers, aged from 21 to 55 (mean 47.4 years) and weighing 55.6$74.3 \mathrm{~kg}$ (mean $67.9 \mathrm{~kg}$ ), gave their written consent to be studied. Blood pressure, and the results of chest $x$-ray examination, electrocardiogram, and a mid-stream specimen of urine were normal in all. Each volunteer attended on four separate occasions at intervals of one week and each time received an injection of lignocaine when fasting. Randomisation ensured that all 10 volunteers received $100 \mathrm{mg}$ and $200 \mathrm{mg}$ of lignocaine using gas-powered injection and that the same dosages were injected into five subjects subcutaneously and into five intramuscularly using a standard syringe and a 23-gauge needle. The site of all injections was the upper and outer quadrant of the gluteus.

Blood was obtained by non-occlusive venepuncture 2,15 , and 30 minutes after injection and every 30 minutes thereafter for $3 \frac{1}{2}$ hours. Lignocaine concentrations in the blood were measured by enzyme immunoassay. ${ }^{2}$

(2) One healthy adult volunteer received two injections of a solution of sodium pertechnetate in saline, the ${ }^{99} \mathrm{~m} \mathrm{TcO}_{4}$ ion having a molecular weight of 163 , into the belly of the extensor muscles of the right forearm, the first by jet injection and the second one week later with a standard syringe and 23-gauge needle. In both cases the injected volume was $0.5 \mathrm{ml}$, containing $3.7 \mathrm{MBq}(100 \mu \mathrm{Ci})$ of radioactivity. Before jet injection the instrument was discharged three times into absorbent material in a laminar flow cabinet to flush the dead space of inactive fluid. An Ohio-Nuclear Series 100 gamma camera was used fitted with a high resolution collimator appropriate to the $140 \mathrm{KeV}$ gamma photon energy of ${ }^{99 \mathrm{~m}} \mathrm{Tc}$. Data were collected on-line by a Varian $620 \mathrm{~L}-100$ computer system, which recorded images at the rate of three frames per minute in a $64 \times 64$ matrix format.

The injection was performed over the face of the gamma camera, the arm and instrument or syringe and needle being enclosed in an absorbent lined polyethylene bag to collect any vapour released during

\section{St Bartholomew's Hospital, London EC1 7BE}

E D COOKE, MD, clinician-in-charge, department of medical electronics SALLY A BOWCOCK, SRN, SCM, research assistant in medical electronics A JOHNSTON, BSC, biochemist in clinical pharmacology

A T ELLIOTT, PHD, MINSTP, principal physicist, department of nuclear medicine and radioisotopes injection. Immediately afterwards the bag and instrument were taken from the camera's field of view and the injected site swabbed. The radioactivity of the bag and swab was then measured. The arm was positioned on the collimator face so that the injection site was tangential to the normal to the collimator face and in the centre of the camera's field of view. Also a single probe comprising a $10-\mathrm{cm}$ diameter sodium iodide crystal fitted with a wide-bore flat-field collimator was positioned behind the subject. The field of view encompassed the region of the heart and excluded the possibility of detecting radioactivity from the injection site. Probe output was fed to a chart recorder and to a paper tape data-logger, the latter reading out the accumulated counts within 10-second intervals. Data collection by the camera/computer system and the probe was started simultaneously with the injection and continued for 25 minutes.

\section{Results}

The accumulated data from the first part of this investigation (figs 1 and 2) show a close comparability between plasma lignocaine concentrations obtained after syringe and needle injection and gaspowered jet injection $\left(\chi^{2}=0.944 ; p<0.001\right)$. The regression coefficient

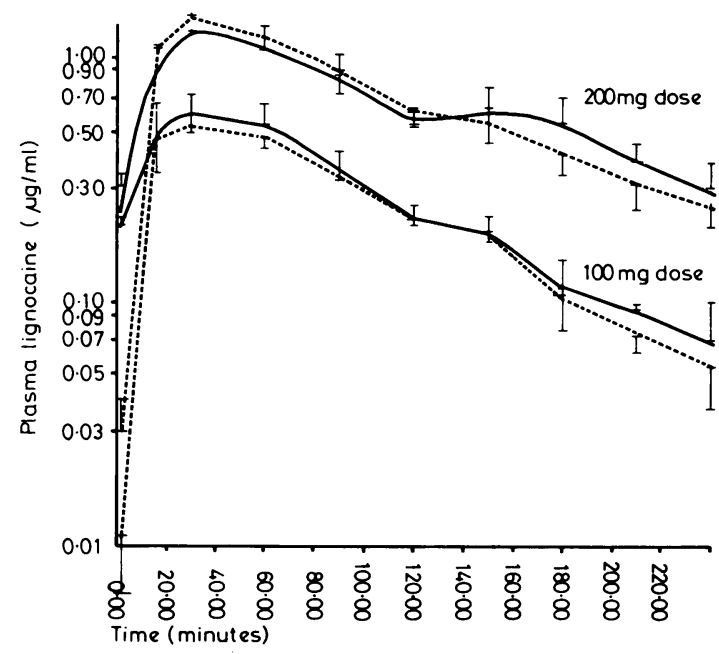

FIG 1-Comparison of plasma lignocaine concentrations obtained using jet-injection (broken line) and intramuscular injection with needle and syringe (continuous line). Plasma lignocaine concentrations are shown to 1 SEM.

(fig 3), plotting the area under the curve (AUC) for conventional intramuscular and subcutaneous injection against delivery by gaspowered jet injection, shows that the slope does not differ from unity $(T=1.01 ; p<0.30)$ and the intercept does not differ from $0(T=0.25$; $\mathrm{p}<0 \cdot 8$ ).

In the second part of the investigation the data obtained from the probe positioned posteriorly over the heart and by summing the counts within the region of interest (ROI) surrounding the injection site in each of the gamma camera images are shown in fig 4 . In each case the ROI data can be well fitted by an experimental curve of the form:

$$
N_{t}=N_{0} e^{-\lambda t}
$$

where $N_{t}$ is the number of counts within the ROI at a time " $t$ " minutes after injection. For the syringe and needle injection, $\lambda$ is approximately $0.09 \mathrm{~min}^{-1}$, corresponding to a clearance half-time of 8 
minutes. The same values for gas-powered injection, $\lambda=0 \cdot 10$, clearance half-time 6.8 minutes, are not significantly different.

With using gas-powered injection the amount of radioactivity found in the polyethylene bag or from the swab of the injection site was less than $3.7 \mathrm{kBq}(0.1 \mu \mathrm{Ci})$; thus the amount of substance injected was greater than $99.8 \%$ of the selected dosage.

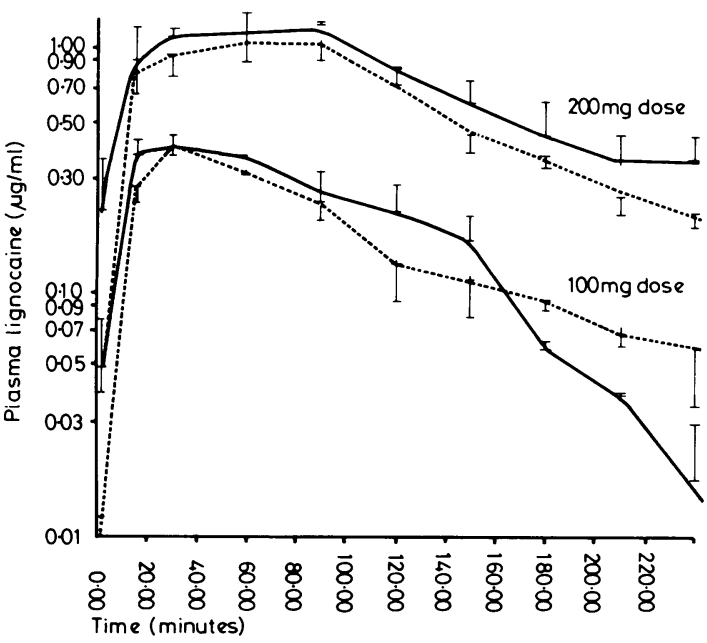

FIG 2-Comparison of plasma lignocaine concentrations obtained using jet-injection (broken line) and subcutaneous injection with needle and syringe (continuous line). Plasma lignocaine concentrations are shown to 1 SEM.

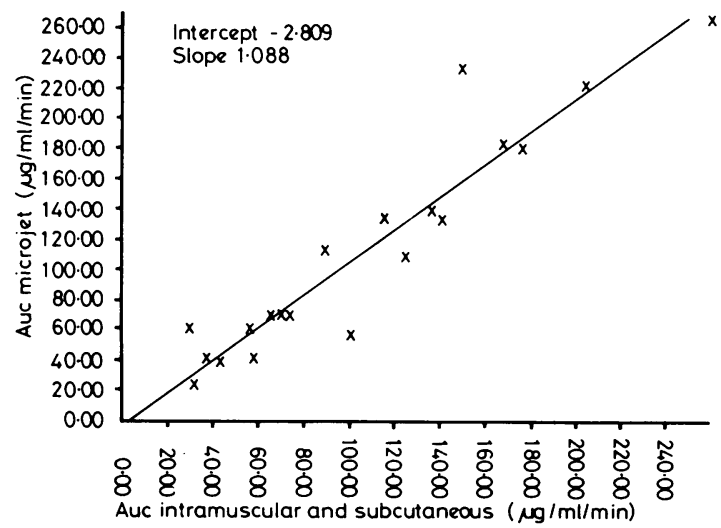

FIG 3-Plotting the area under the curve (AUC) of plasma lignocaine levels obtained by conventional methods against jet injection shows that the slope is not significantly different from unity and intercept does not differ from zero.

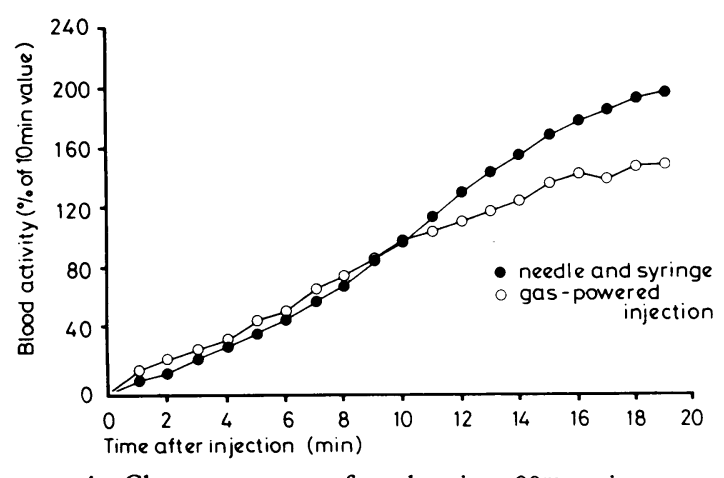

FIG 4-Clearance rates of technetium-99m using gaspowered injection and needle and syringe.

\section{Discussion}

This investigation has shown that there is a close correlation between plasma concentrations of lignocaine when delivered by gas-powered injection as compared with conventional methods. This result would be expected from the values obtained from the study of clearance half time. Moreover, dosage, as judged by residual radioactivity of the surrounding air and of a swab obtained from the skin immediately after injection, appears to be highly accurate - that is, there was no splash-back phenomenon from the injection site.

After jet injection the substance is dispersed in the tissuess (E D Cooke and S A Bowcock, unpublished data), rather than pooled. This may account for the slight divergence in plasma concentrations and clearance rates that occurred between 10 and 12 minutes after the injection, and that has been observed before using insulin. ${ }^{4}$ This is unlikely to be clinically relevant, however. Thus our results suggest that gas-powered jet injection may be used with confidence for administering the wide range of therapeutic substances that must be given by injection. Asepsis after multiple injections has been previously shown. ${ }^{1}$

The cost of a Med-E-Jet system is about $£ 650$, but the real cost per injection, according to the manufacturers, drops after several thousand injections to $1 \mathrm{p}$ compared with $7 \mathrm{p}$ for needle and syringe.

Financial support for this investigation was provided by Weddle Pharmaceutical Ltd.

\section{References}

${ }^{1}$ Black J, Nagle CD, Strachan CJL. Prophylactic low-dose heparin by jet injection. Br Med F 1978;ii:95-6.

2 Juin S, Johnston A. The measurement of lignocaine at low concentrations in plasma, a comparison of gas liquid chromatography with enzyme immunoassay. Br $\mathcal{f}$ Clin Pharmacol 1979;8:598-9.

${ }^{3}$ Cohn L, Chez RA, Hingson RA, Szulman AE, Trimmer M. Use of jet insulin injection in diabetes mellitus therapy. Diabetes $1972 ; 21: 39-44$

4 Cohn L, Hingson RA, Narduzzi JV, Seddon JM. Clinical experience with jet insulin injection in diabetes mellitus therapy: a clue to the pathogenesis of lipodystrophy. Ala F Med Sec 1974;11:265-73.

(Accepted 22 fuly 1980)

ONE HUNDRED YEARS AGO The question of real or apparent death having recently come before me in a few cases, in which it would have been difficult for anyone but a medical jurist to give a scientific or confident answer, it occurred to me that a sign of death which is so very simple in its application, and as far as I am aware not generally known, or at any rate rarely put to the test, would not be unacceptable to some of one's professional brethren, as one worth committing to memory. I refer to a condition of complete flaccidity of the iris in real death. It can easily be shown by synchronous compression of the globe of the eye in two opposite directions, when the pupil will readily assume an oval or irregular shape, whereas in cases of apparent death no ordinary amount of compression in this manner will have the least effect in altering the usual circular form of the pupil. I have recently made several observations in cases of suspended animation, of coma, and of impending death from various causes; also shortly after death; and in every case with success, as regards confirming the test. This sign of death was first pointed out to me by Sir William Jenner; and as I had never seen it in print I thought it was an original observation, but on looking up the literature of the subject, I find the fact mentioned first by $M$ Ripault. However, in every case in which I have tested the sign it has turned out so uniformly reliable, that I venture to think it a valuable help in determining the question of real and apparent death, especially as it is one which can be attested before the cooling of the body, and before the supervention of rigor mortis, and considering that life is not incompatible with a temporary suspension of the important functions of respiration and circulation. BOYD B JoLL, MBLond. (British Medical fournal, 1880.) 